Communications in Physics, Vol.18, No. 1 (2008), pp. 9-18

\title{
NEUTRINOLESS DOUBLE BETA DECAY IN THE ECONOMICAL 3-3-1 MODEL
}

\author{
DANG VAN SOA, NGUYEN HUY THAO \\ Department of Physics, Hanoi University of Education, Hanoi, Vietnam \\ PHUNG VAN DONG, TRINH THI HUONG, AND HOANG NGOC LONG \\ Institute of Physics, VAST, P. O. Box 429, Bo Ho, Hanoi 10000, Vietnam
}

\begin{abstract}
Possible contributions to neutrinoless double beta $(\beta \beta)_{0 \nu}$ decay in the economical 3-3-1 model are discussed. We show that the $(\beta \beta)_{0 \nu}$ decay in this model is due to both sourcesMajorana $\left\langle M_{\nu}\right\rangle_{L}$ and Dirac $\left\langle M_{\nu}\right\rangle_{D}$ neutrino masses. If the mixing angle between charged gauge bosons, the standard model $W$ and bilepton $Y$ is in range of the ratio of neutrino masses $\left\langle M_{\nu}\right\rangle_{L} /\left\langle M_{\nu}\right\rangle_{D}$, then both the Majorana and Dirac masses simultaneously give contributions dominant to the decay. As results, constraints on the bilepton mass are also given.
\end{abstract}

\section{INTRODUCTION}

In the standard model (SM) of strong and electroweak interactions, the neutrinos are strictly massless due to absence of right-handed chiral states $\left(\nu_{R}\right)$ and requirement of $\mathrm{SU}(2)_{L} \otimes \mathrm{U}(1)_{Y}$ gauge invariance and renormalizability. Recent experimental results of SuperKamiokande Collaboration [1], KamLAND [2] and SNO [3] confirm that the neutrinos have tiny masses and oscillate, this implies that the SM must be extended. Among beyond-SM extensions, the models based on $\mathrm{SU}(3)_{C} \otimes \mathrm{SU}(3)_{L} \otimes \mathrm{U}(1)_{X}(3-3-1)$ gauge group $[4,5]$ have some intriguing features: First, they can give partial explanation of the generation number problem. Second, the third quark generation has to be different from the first two, so this leads to possible explanation of why top quark is uncharacteristically heavy.

In one of 3-3-1 models three lepton triplets are of the form $\left(\nu_{L}, l_{L}, \nu_{R}^{c}\right)$ and the scalar sector is minimal with just two Higgs triplets, hence it has been called the economical 3-3-1 model [6]. The general Higgs sector is very simple and consists of three physical scalars (two neutral and one charged) and eight Goldstone bosons - the needed number for massive gauge bosons [7]. The model is consistent and possesses key properties: (i) There are three quite different scales of vacuum expectation values (VEVs): $u \sim O(1) \mathrm{GeV}$, $v \approx 246 \mathrm{GeV}$, and $\omega \sim \mathcal{O}(1) \mathrm{TeV}$; (ii) There exist two types of Yukawa couplings with very different strengths, the lepton-number conserving (LNC) $h$ 's and the lepton-number violating (LNV) $s$ 's, satisfying $s \ll h$. The resulting model yields interesting physical phenomenologies due to mixings in the Higgs [7], gauge [8] and quark [9] sectors.

Despite present experimental advances in neutrino physics, we have not yet known if the neutrinos are Dirac or Majorana particles. If the neutrinos are Majorana ones, the mass terms violate lepton number by two units, which may result in important consequences in particle physics and cosmology. A crucial process that will help in determining neutrino 
nature is the neutrinoless double beta $(\beta \beta)_{0 \nu}$ decay [10]. It is also a typical process which requires violation of the lepton number, although it could say nothing about the value of the mass. This is because although right-handed currents and/or scalar bosons may affect the decay rate, it has been shown that whatever the mechanism of this decay is a nonvanishing neutrino mass [11]. In some models $(\beta \beta)_{0 \nu}$ decay can proceed with arbitrary small neutrino mass via scalar boson exchange [12].

The mechanism involving a trilinear interaction of the scalar bosons was proposed in Ref. [13] in the context of model with $\mathrm{SU}(2) \otimes \mathrm{U}(1)$ symmetry with doublets and a triplet of scalar bosons. However, since in these types of models there is no large mass scale [14], the contribution of the trilinear interaction is, in fact, negligible. In general, in models with that symmetry, a fine tuning is needed if we want the trilinear terms to give important contributions to the $(\beta \beta)_{0 \nu}$ decay [15]. It was shown in Ref. $[16,17]$ that in $3-3-1$ models, which has a rich Higgs bosons sector, there are new many contributions to the $(\beta \beta)_{0 \nu}$ decay. In recent work [18], authors showed that the implementation of spontaneous breaking of the lepton number in the 3-3-1 model with right-handed neutrinos gives rise to fast neutrino decay with Majoron emission and generates a bunch of new contributions to the $(\beta \beta)_{0 \nu}$ decay.

In an earlier work [19] we have analyzed the neutrino masses in the economical 3-3-1 model. The masses of neutrinos are given by three different sources widely ranging over the mass scales including the GUT's and the small VEV $u$ of spontaneous lepton breaking. With a finite renormalization in mass, the spectrum of neutrino masses is neat and can fit the data. In this work, we will discuss possible contributions to the $(\beta \beta)_{0 \nu}$ decay in the considering model. We show that in contradiction with previous analysis, the $(\beta \beta)_{0 \nu}$ decay arises from two different sources, which require both the non-vanishing Majorana and Dirac neutrino masses. If the mixing angle between the charged gauge bosons is in range of the ratio of neutrino masses $\left\langle M_{\nu}\right\rangle_{L} /\left\langle M_{\nu}\right\rangle_{D}$, both the Majorana and Dirac masses simultaneously give dominant contributions to the decay. Based on the results, the constraints on the bilepton mass are also given.

The rest of this paper is organized as follows: In Section II we give a brief review of the economical 3-3-1 model, in which a new bound of the mixing angle is given from $Z$ decay into neutrinos, which violates the lepton number. Section III is devoted to detailed analysis of the possible contributions to the $(\beta \beta)_{0 \nu}$ decay. We summarize our results and make conclusions in the last section - Sec. IV.

\section{A REVIEW OF THE MODEL}

The particle content in this model which is anomaly free is given as follows [6]

$$
\begin{aligned}
& \psi_{a L}=\left(\nu_{a L}, l_{a L},\left(\nu_{a R}\right)^{c}\right)^{T} \sim(3,-1 / 3), \quad l_{a R} \sim(1,-1), \quad a=1,2,3, \\
& Q_{1 L}=\left(u_{1 L}, d_{1 L}, U_{L}\right)^{T} \sim(3,1 / 3), \quad Q_{\alpha L}=\left(d_{\alpha L},-u_{\alpha L}, D_{\alpha L}\right)^{T} \sim\left(3^{*}, 0\right), \quad \alpha=2,3, \\
& u_{a R} \sim(1,2 / 3), \quad d_{a R} \sim(1,-1 / 3), \quad U_{R} \sim(1,2 / 3), \quad D_{\alpha R} \sim(1,-1 / 3),
\end{aligned}
$$

where the values in the parentheses denote quantum numbers based on the $\left(\mathrm{SU}(3)_{L}, \mathrm{U}(1)_{X}\right)$ symmetry. Unlike the usual 3-3-1 model with right-handed neutrinos, where the third family of quarks should be discriminating, in the model under consideration the first family 
has to be different from the two others [9]. The electric charge operator in this case takes a form

$$
Q=T_{3}-\frac{1}{\sqrt{3}} T_{8}+X,
$$

where $T_{i}(i=1,2, \ldots, 8)$ and $X$, respectively, stand for $\mathrm{SU}(3)_{L}$ and $\mathrm{U}(1)_{X}$ charges. The electric charges of the exotic quarks $U$ and $D_{\alpha}$ are the same as of the usual quarks, i.e., $q_{U}=2 / 3, q_{D_{\alpha}}=-1 / 3$.

The spontaneous symmetry breaking in this model is obtained by two stages:

$$
\mathrm{SU}(3)_{L} \otimes \mathrm{U}(1)_{X} \rightarrow \mathrm{SU}(2)_{L} \otimes \mathrm{U}(1)_{Y} \rightarrow \mathrm{U}(1)_{Q}
$$

The first stage is achieved by a Higgs scalar triplet with a VEV given by

$$
\chi=\left(\chi_{1}^{0}, \chi_{2}^{-}, \chi_{3}^{0}\right)^{T} \sim(3,-1 / 3), \quad\langle\chi\rangle=\frac{1}{\sqrt{2}}(u, 0, \omega)^{T} .
$$

The last stage is achieved by another Higgs scalar triplet needed with the VEV as follows

$$
\phi=\left(\phi_{1}^{+}, \phi_{2}^{0}, \phi_{3}^{+}\right)^{T} \sim(3,2 / 3), \quad\langle\phi\rangle=\frac{1}{\sqrt{2}}(0, v, 0)^{T} .
$$

The Yukawa interactions which induce masses for the fermions can be written in the most general form:

$$
\mathcal{L}_{\mathrm{Y}}=\mathcal{L}_{\mathrm{LNC}}+\mathcal{L}_{\mathrm{LNV}}
$$

in which, each part is defined by

$$
\begin{aligned}
\mathcal{L}_{\mathrm{LNC}}= & h^{U} \bar{Q}_{1 L \chi} \chi U_{R}+h_{\alpha \beta}^{D} \bar{Q}_{\alpha L} \chi^{*} D_{\beta R} \\
& +h_{a b}^{l} \bar{\psi}_{a L} \phi l_{b R}+h_{a b}^{\nu} \epsilon_{p m n}\left(\bar{\psi}_{a L}^{c}\right)_{p}\left(\psi_{b L}\right)_{m}(\phi)_{n} \\
& +h_{a}^{d} \bar{Q}_{1 L} \phi d_{a R}+h_{\alpha a}^{u} \bar{Q}_{\alpha L} \phi^{*} u_{a R}+H . c ., \\
\mathcal{L}_{\mathrm{LNV}}= & s_{a}^{u} \bar{Q}_{1 L} \chi u_{a R}+s_{\alpha a}^{d} \bar{Q}_{\alpha L} \chi^{*} d_{a R} \\
& +s_{\alpha}^{D} \bar{Q}_{1 L} \phi D_{\alpha R}+s_{\alpha}^{U} \bar{Q}_{\alpha L} \phi^{*} U_{R}+\text { H.c. },
\end{aligned}
$$

where $p, m$ and $n$ stand for $\mathrm{SU}(3)_{L}$ indices.

The VEV $\omega$ gives mass for the exotic quarks $U, D_{\alpha}$ and the new gauge bosons $Z^{\prime}, X, Y$, while the VEVs $u$ and $v$ give mass for all the ordinary fermions and gauge bosons $[9,19]$. To keep a consistency with the effective theory, the VEVs in this model have to satisfy the constraint

$$
u^{2} \ll v^{2} \ll \omega^{2} .
$$

In addition we can derive $v \approx v_{\text {weak }}=246 \mathrm{GeV}$ and $|u| \leq 2.46 \mathrm{GeV}$ from the mass of $W$ boson and the $\rho$ parameter [6], respectively. From atomic parity violation in cesium, the bound for the mass of new natural gauge boson is given by $M_{Z^{\prime}}>564 \mathrm{GeV}(\omega>1400 \mathrm{GeV})$ [8]. From the analysis on quark masses, higher values for $\omega$ can be required, for example, up to $10 \mathrm{TeV}[9]$.

The Yukawa couplings of (7) possess an extra global symmetry [20] not broken by $v, \omega$ but by $u$. From these couplings, one can find the following lepton symmetry $L$ as in Table 1 (only the fields with nonzero $L$ are listed; all other ones have vanishing $L$ ). Here $L$ is broken by $u$ which is behind $L\left(\chi_{1}^{0}\right)=2$, i.e., $u$ is a kind of the SLB scale [21]. 
Table 1. Nonzero lepton number $L$ of the model particles.

\begin{tabular}{ccccccccc} 
Field & $\nu_{a L}$ & $l_{a L, R}$ & $\nu_{a R}^{c}$ & $\chi_{1}^{0}$ & $\chi_{2}^{-}$ & $\phi_{3}^{+}$ & $U_{L, R}$ & $D_{\alpha L, R}$ \\
\hline$L$ & 1 & 1 & -1 & 2 & 2 & -2 & -2 & 2
\end{tabular}

It is interesting that the exotic quarks also carry the lepton number; therefore, this $L$ obviously does not commute with the gauge symmetry. One can then construct a new conserved charge $\mathcal{L}$ through $L$ by making a linear combination $L=x T_{3}+y T_{8}+\mathcal{L} I$. Applying $L$ on a lepton triplet, the coefficients will be determined

$$
L=\frac{4}{\sqrt{3}} T_{8}+\mathcal{L} I
$$

Another useful conserved charge $\mathcal{B}$ exactly not broken by $u, v$ and $\omega$ is usual baryon number $B=\mathcal{B} I$. Both the charges $\mathcal{L}$ and $\mathcal{B}$ for the fermion and Higgs multiplets are listed in Table 2 .

Table 2. $\quad \mathcal{B}$ and $\mathcal{L}$ charges of the model multiplets.

\begin{tabular}{ccccccccccc} 
Multiplet & $\chi$ & $\phi$ & $Q_{1 L}$ & $Q_{\alpha L}$ & $u_{a R}$ & $d_{a R}$ & $U_{R}$ & $D_{\alpha R}$ & $\psi_{a L}$ & $l_{a R}$ \\
\hline $\mathcal{B}$-charge & 0 & 0 & $\frac{1}{3}$ & $\frac{1}{3}$ & $\frac{1}{3}$ & $\frac{1}{3}$ & $\frac{1}{3}$ & $\frac{1}{3}$ & 0 & 0 \\
\hline $\mathcal{L}$-charge & $\frac{4}{3}$ & $-\frac{2}{3}$ & $-\frac{2}{3}$ & $\frac{2}{3}$ & 0 & 0 & -2 & 2 & $\frac{1}{3}$ & 1
\end{tabular}

Let us note that the Yukawa couplings of $(8)$ conserve $\mathcal{B}$, however, violate $\mathcal{L}$ with \pm 2 units which implies that these interactions are much smaller than the first ones [9]:

$$
s_{a}^{u}, s_{\alpha a}^{d}, s_{\alpha}^{D}, s_{\alpha}^{U} \ll h^{U}, h_{\alpha \beta}^{D}, h_{a}^{d}, h_{\alpha a}^{u} .
$$

A consequence of $u \neq 0$ is that the standard model gauge boson $W^{\prime}$ and bilepton $Y^{\prime}$ $\operatorname{mix}$

$$
\mathcal{L}_{\text {mass }}^{\mathrm{CG}}=\frac{g^{2}}{4}\left(W^{\prime-}, Y^{\prime-}\right)\left(\begin{array}{cc}
u^{2}+v^{2} & u \omega \\
u \omega & \omega^{2}+v^{2}
\end{array}\right)\left(\begin{array}{c}
W^{\prime+} \\
Y^{\prime+}
\end{array}\right) .
$$

Physical charged gauge bosons are given by

$$
\begin{aligned}
W & =\cos \theta W^{\prime}+\sin \theta Y^{\prime}, \\
Y & =-\sin \theta W^{\prime}+\cos \theta Y^{\prime},
\end{aligned}
$$

where the mixing angle is

$$
\tan \theta=\frac{u}{\omega} .
$$

There exist LNV terms in the charged currents proportional to $\sin \theta$

$$
H^{\mathrm{CC}}=\frac{g}{\sqrt{2}}\left(J_{W}^{\mu+} W_{\mu}^{-}+J_{Y}^{\mu+} Y_{\mu}^{-}+\text {H.c. }\right)
$$


with

$$
\begin{aligned}
J_{W}^{\mu+}= & c_{\theta}\left(\bar{l}_{a L} \gamma^{\mu} \nu_{a L}+\bar{d}_{a L} \gamma^{\mu} u_{a L}\right) \\
& -s_{\theta}\left(\bar{l}_{a L} \gamma^{\mu} \nu_{a R}^{c}+\bar{d}_{1 L} \gamma^{\mu} U_{L}+\bar{D}_{\alpha L} \gamma^{\mu} u_{\alpha L}\right) \\
J_{Y}^{\mu+}= & c_{\theta}\left(\bar{l}_{a L} \gamma^{\mu} \nu_{a R}^{c}+\bar{d}_{1 L} \gamma^{\mu} U_{L}+\bar{D}_{\alpha L} \gamma^{\mu} u_{\alpha L}\right) \\
& +s_{\theta}\left(\bar{l}_{a L} \gamma^{\mu} \nu_{a L}+\bar{d}_{a L} \gamma^{\mu} u_{a L}\right)
\end{aligned}
$$

As in Ref. [6], the constraint on the $W-Y$ mixing angle $\theta$ from the $W$ width is given by $\sin \theta \leq 0.08$. However, in the following we will show that a more stricter bound can obtain from the invisible $Z$ width through the unnormal neutral current of LNV:

$$
\begin{aligned}
\mathcal{L}_{\text {unnormal }}^{\mathrm{NC}=} & -\frac{g t_{2 \theta} g_{k V}(\nu)}{c_{W}}\left(\bar{\nu}_{a L} \gamma^{\mu} \nu_{a R}^{c}+\bar{u}_{1 L} \gamma^{\mu} U_{L}\right. \\
& \left.-\bar{D}_{\alpha L} \gamma^{\mu} d_{\alpha L}\right) Z_{\mu}^{k}+H . c .
\end{aligned}
$$

where the neutrino coupling constants $\left(g_{k V}, k=1,2\right)$ are given by

$$
\begin{aligned}
g_{1 V}\left(\nu_{L}\right) & \simeq \frac{c_{\varphi}-s_{\varphi} \sqrt{4 c_{W}^{2}-1}}{2} \\
g_{2 V}\left(\nu_{L}\right) & \simeq \frac{s_{\varphi}+c_{\varphi} \sqrt{4 c_{W}^{2}-1}}{2} .
\end{aligned}
$$

Let us note that the LNV interactions mediated by neutral gauge bosons $Z^{1}$ and $Z^{2}$ exist only in the neutrino and exotic quark sectors.

The interactions in (17) for the neutrinos lead to additional invisible-decay modes for the $Z$ boson. For each generation of lepton, the corresponding invisible-decay width gets approximation:

$$
\Gamma_{\nu_{L} \nu_{R}} \simeq \frac{1}{2} t_{2 \theta}^{2} \Gamma_{\nu_{L} \nu_{L}}^{\mathrm{SM}}
$$

where $\Gamma_{\nu_{L} \nu_{L}}^{\mathrm{SM}}=\frac{G_{F} M_{Z}^{3}}{12 \pi \sqrt{2}}$ is the SM prediction for the decay rate of $Z$ into a pair of neutrinos; $\varphi$ and $\theta$ take small values. The experimental data for the total invisible neutrino decay modes give us [22]

$$
\Gamma_{\text {invi }}^{\text {exp }}=(2.994 \pm 0.012) \Gamma_{\nu_{L} \nu_{L}}^{\mathrm{SM}} .
$$

From (20) and (21) we get an upper limit for the mixing angle

$$
t_{\theta} \leq 0.03
$$

which is smaller than that given in Ref. [6]. 


\section{THE NEUTRINOLESS DOUBLE BETA DECAY}

The interactions that lead to the $(\beta \beta)_{0 \nu}$ decay involve hadrons and leptons. For the case of the standard contribution, its amplitude can be written as [18]

$$
M_{(\beta \beta)_{0 \nu}}=\frac{g^{4}}{4 m_{W}^{4}} M_{\mu \nu}^{h} \bar{u} \gamma^{\mu} P_{L} \frac{\not 1+m_{\nu}}{q^{2}-m_{\nu}^{2}} \gamma^{\nu} P_{R} v
$$

with $M_{\mu \nu}^{h}$ carrying the hadronic information of the process and $P_{R, L}=\frac{\left(1 \pm \gamma_{5}\right)}{2}$. In the presence of neutrino mixing and considering that $m_{\nu}^{2} \ll q^{2}$, we can write

$$
M_{(\beta \beta)_{0 \nu}}=A_{(\beta \beta)_{0 \nu}} M_{\mu \nu}^{h} \bar{u} P_{R} \gamma^{\mu} \gamma^{\nu} v,
$$

where

$$
A_{(\beta \beta)_{0 \nu}}=\frac{g^{4}\left\langle M_{\nu}\right\rangle}{4 m_{W}^{4}\left\langle q^{2}\right\rangle}
$$

is the strength of effective coupling of the standard contribution. For the case of three neutrino species $\left\langle M_{\nu}\right\rangle=\sum U_{e i}^{2} m_{\nu}$ is the effective neutrino mass which we use as reference value $0.2 \mathrm{eV}$ and $\left\langle q^{2}\right\rangle$ is the average of the transferred squared four-momentum.

The contributions to the $(\beta \beta)_{0 \nu}$ decay in our model coming from the charged gauge bosons $W^{-}$and $Y^{-}$dominate the process. As the $(\beta \beta)_{0 \nu}$ decay has not been experimentally detected yet, the analysis we do here is to obtain the new contributions and to compare them with the standard one $[11,17]$. For the standard contribution as depicted in Fig. 1.a), its effective coupling takes the form

$$
A_{(\beta \beta)_{0 \nu}}(1 . a)=\frac{g^{4}\left\langle M_{\nu}\right\rangle_{L}}{4 m_{W}^{4}\left\langle q^{2}\right\rangle} c_{\theta}^{4},
$$

where $M_{L}$ is the Majorana mass. The first new contribution involves only $W^{-}$as of the standard one, but now interacts with two charged currents $J_{\mu}$ and $J_{\mu}^{c}$ as depicted in Fig. (1.b). It is to be noted that in this case the Dirac mass gives the contribution to the effective coupling

$$
A_{(\beta \beta)_{0 \nu}}(1 . b)=\frac{g^{4}\left\langle M_{\nu}\right\rangle_{D}}{4 m_{W}^{4}\left\langle q^{2}\right\rangle} c_{\theta}^{3} s_{\theta}
$$

where $M_{D}$ is the Dirac mass.

From Eqs. (26) and (27) we see that the LNV in the $(\beta \beta)_{0 \nu}$ decay arises from two different sources identified by the non-vanishing Majorana and Dirac mass terms, respectively. In Fig. (1.a) the LNV is due to the Majorana mass, while that in Fig. (1.b) is by the LNV coupling of $W$ boson to the charged current (the term is proportional to $\sin \theta$ ). In comparing both effective couplings, we obtain the ratio

$$
\frac{A_{(\beta \beta)_{0 \nu}}(1 . b)}{A_{(\beta \beta)_{0 \nu}}(1 . a)}=\frac{\left\langle M_{\nu}\right\rangle_{D}}{\left\langle M_{\nu}\right\rangle_{L}} \tan \theta
$$

From (28) we see that the relevance of this contribution depends on angle $\theta$ and also the ratio between $\left\langle M_{\nu}\right\rangle_{D}$ and $\left\langle M_{\nu}\right\rangle_{L}$. It is worth noting that if $\left\langle M_{\nu}\right\rangle_{D} \cdot t_{\theta} \sim\left\langle M_{\nu}\right\rangle_{L}$ then both 


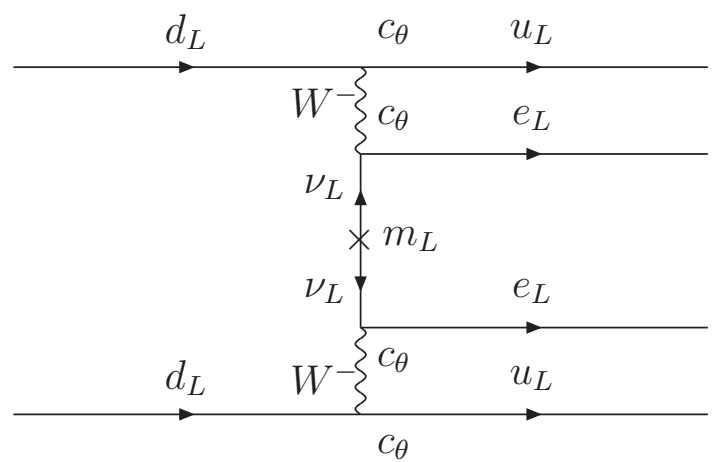

(a)

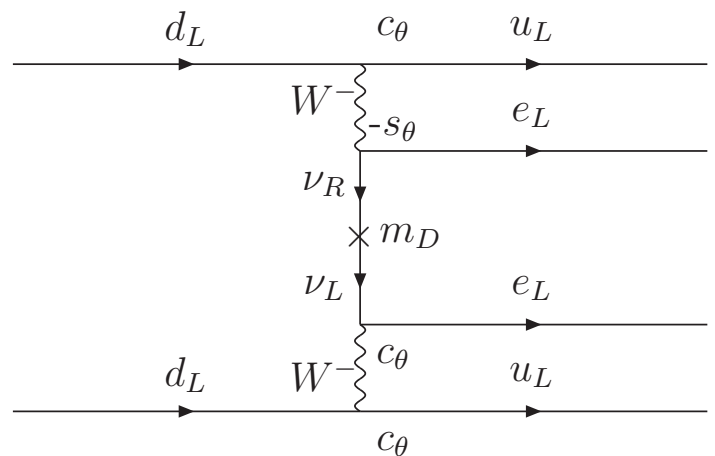

(b)

Fig. 1. Contribution of the SM bosons to the $(\beta \beta)_{0 \nu}$ decay.

Majorana and Dirac masses simultaneously give the dominant contributions to the $(\beta \beta)_{0 \nu}$ decay.

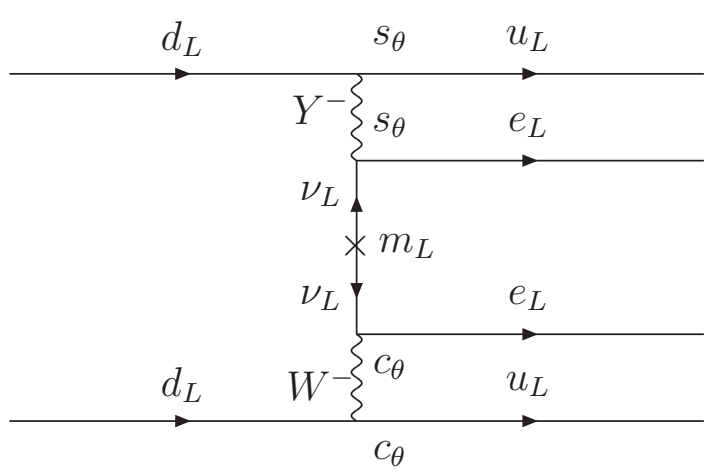

(a)

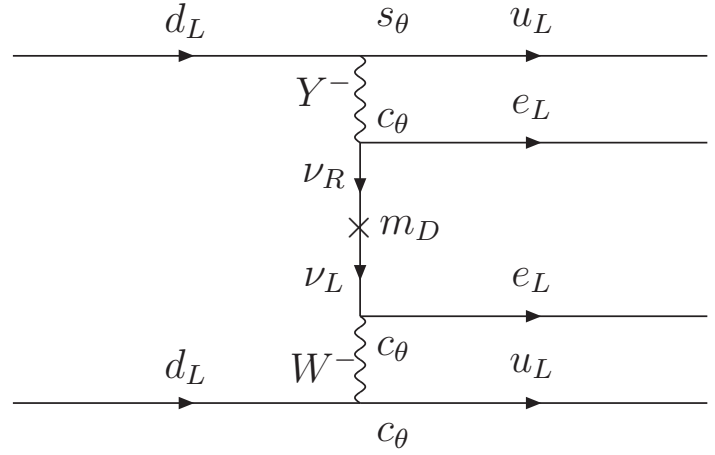

(b)

Fig. 2. Contribution of both the $\mathrm{W}$ and $\mathrm{Y}$ to the $(\beta \beta)_{0 \nu}$ decay.

Next, we consider contributions that involve both $W^{-}$and $Y^{-}$. It involves the two currents $J_{\mu}$ and $J_{\mu}^{c}$ interacting with $W$ and $Y$, as depicted in Fig. (2.a) for $\left\langle M_{\nu}\right\rangle_{L}$ and Fig.(2.b) for $\left\langle M_{\nu}\right\rangle_{D}$. The effective couplings in this case are

$$
A_{(\beta \beta)_{0 \nu}}(2 . a)=\frac{g^{4}\left\langle M_{\nu}\right\rangle_{L} c_{\theta}^{2} s_{\theta}^{2}}{4 m_{W}^{2} m_{Y}^{2}\left\langle q^{2}\right\rangle},
$$

and

$$
A_{(\beta \beta)_{0 \nu}}(2 . b)=\frac{g^{4}\left\langle M_{\nu}\right\rangle_{D} c_{\theta}^{3} s_{\theta}}{4 m_{W}^{2} m_{Y}^{2}\left\langle q^{2}\right\rangle}
$$


From (29) and (30) we see that the case with the Majorana mass gives the contribution to the $(\beta \beta)_{0 \nu}$ much smaller than the Dirac one. Comparing with the standard effective coupling, we get the ratios

$$
\frac{A_{(\beta \beta)_{0 \nu}}(2 . b)}{A_{(\beta \beta)_{0 \nu}}(1 . a)}=\left(\frac{m_{W}^{2}}{m_{Y}^{2}}\right) \frac{\left\langle M_{\nu}\right\rangle_{D}}{\left\langle M_{\nu}\right\rangle_{L}} \tan \theta,
$$

and

$$
\frac{A_{(\beta \beta)_{0 \nu}}(2 . a)}{A_{(\beta \beta)_{0 \nu}}(1 . a)}=\left(\frac{m_{W}^{2}}{m_{Y}^{2}}\right) \tan ^{2} \theta .
$$

Differing from the previous case, Eq.(31) shows that the relevance of these contributions depends on the angle $\theta$, the ratio $\frac{\left\langle M_{\nu}\right\rangle_{D}}{\left\langle M_{\nu}\right\rangle_{L}}$ and the bilepton mass also. Suppose that the new contributions are smaller than the standard one, from Eq. (31) we get a lower bound for the bilepton mass

$$
m_{Y}^{2}>m_{W}^{2} \frac{\left\langle M_{\nu}\right\rangle_{D}}{\left\langle M_{\nu}\right\rangle_{L}} \tan \theta
$$

Taking $m_{W}^{2}=80.425 G e V, t_{\theta}=0.03$, the low bounds of mass $m_{Y}$ in range of $\frac{\left\langle M_{\nu}\right\rangle_{D}}{\left\langle M_{\nu}\right\rangle_{L}} \sim$ $10^{2}-10^{3}[19]$ are given in Table III. It is interesting to note that from "wrong " muon

Table 3. The low bound of bilepton mass in range of $\frac{\left\langle M_{\nu}\right\rangle_{D}}{\left\langle M_{\nu}\right\rangle_{L}}$

$\begin{array}{lrrrrrr}\frac{\left\langle M_{\nu}\right\rangle_{D}}{\left\langle M_{\nu}\right\rangle_{L}} & 100 & 200 & 400 & 600 & 800 & 1000 \\ m_{Y}(G e V) & 139.0 & 197.0 & 278.6 & 341.2 & 394.0 & 440.5\end{array}$

decay experiments one obtains a bound for the bilepton mass : $m_{Y} \geq 230 \mathrm{GeV}$ [27]. From Eq. (32) we see that the order of contribution is much smaller than standard contribution, this is due to the LNV in the $(\beta \beta)_{0 \nu}$ decay arising from the Majorana mass term and the LNV coupling between the bilepton $Y$ and the charged current $J^{\mu}$ of ordinary quarks and leptons. Taking $m_{Y}=139 \mathrm{GeV}$ we obtain

$$
\frac{A_{(\beta \beta)_{0 \nu}}(2 . a)}{A_{(\beta \beta)_{0 \nu}}(1 . a)} \leq 3.0 \times 10^{-4}
$$

Now we examine the next four contributions which involve only the bileptons $Y$. In Fig. (3.a) we display and example of this kind of contribution where the current $J_{\mu}^{c}$ appears in the two vertices. The effective coupling is

$$
A_{(\beta \beta)_{0 \nu}}(3 . a)=\frac{g^{4}\left\langle M_{\nu}\right\rangle_{L} s_{\theta}^{4}}{4 m_{Y}^{4}\left\langle q^{2}\right\rangle} .
$$

For another case we have also

$$
A_{(\beta \beta)_{0 \nu}}(3 . b)=\frac{g^{4}\left\langle M_{\nu}\right\rangle_{D} c_{\theta} s_{\theta}^{3}}{4 m_{Y}^{4}\left\langle q^{2}\right\rangle} .
$$


Comparing with the standard effective coupling, we get

$$
\frac{A_{(\beta \beta)_{0 \nu}}(3 . a)}{A_{(\beta \beta)_{0 \nu}}(1 . a)}=\left(\frac{m_{W}}{m_{Y}}\right)^{4} \tan ^{4} \theta .
$$

Using the above data, the ratio gets an upper limit

$$
\frac{A_{(\beta \beta)_{0 \nu}}(3 . a)}{A_{(\beta \beta)_{0 \nu}}(1 . a)} \leq 9.0 \times 10^{-8}
$$

which is very small. It is easy to check that the remaining contributions are much smaller than those with the charged $W$ bosons. This is due to the fact that all the couplings of the bilepton with ordinary quarks and leptons in the diagrams of Fig. (3) are LNV.

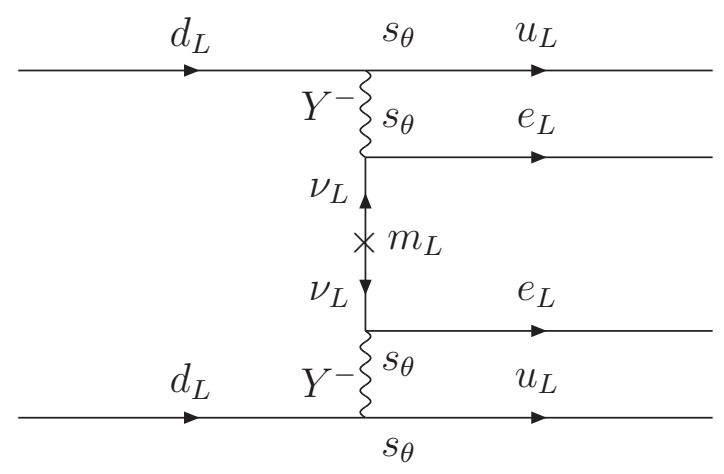

(a)

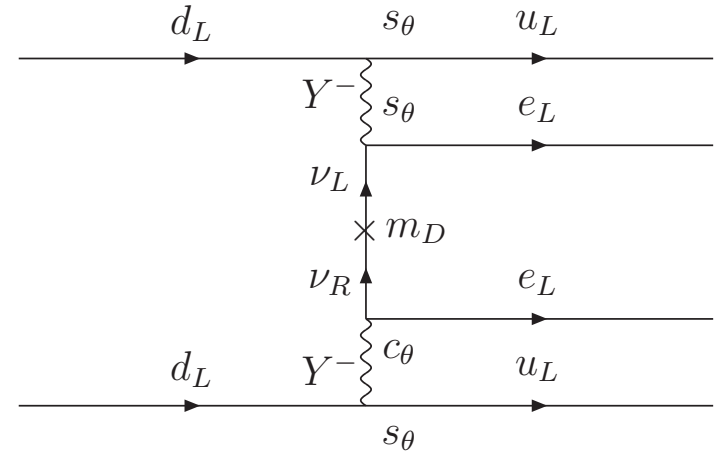

(b)

Fig. 3. Contribution of the bileptons to the $(\beta \beta)_{0 \nu}$ decay.

\section{CONCLUSION}

In this paper we have investigated the implications of spontaneous breaking of the lepton number in the economical 3-3-1 model in the $(\beta \beta)_{0 \nu}$ decay. We have performed a systematic analysis of the couplings of all possible contributions of charged gauge bosons to the decay. The result shows that, the $(\beta \beta)_{0 \nu}$ decay mechanism in the economical 3-3-1 model requires both the non-vanishing Majorana and Dirac masses. If the mixing angle between the charged gauge boson and bilepton is in range of the ratio of neutrino masses $\left\langle M_{\nu}\right\rangle_{L}$ and $\left\langle M_{\nu}\right\rangle_{D}$ then both the Majorana and Dirac masses simultaneously give the dominant contributions to the decay. Based on the result, the constraints on the bilepton mass are given. It is interesting to note that the relevance of the new contributions are dictated by the mixing angle $\theta$, the effective mass of neutrino and the bilepton mass. By estimating the order of magnitude of the new contributions, we predicted that the most robust one is that depicted in Fig. 2 whose order of magnitude is $3.0 \times 10^{-4}$ of the standard contribution. 
DANG VAN SOA et al.

\section{REFERENCES}

[1] SuperKamiokande Collaboration, Y. Fukuda et al., Phys. Rev. Lett. 81 (1998) 1158; 81 (1998) 1562; 82 (1999) 2644; 85 (2000) 3999; Y. Suzuki, Nucl. Phys. B, Proc. Suppl. 77 (1999) 35; S. Fukuda et al., Phys. Rev. Lett. 86 (2001) 5651; Y. Ashie et al., Phys. Rev. Lett. 93 (2004) 101801.

[2] KamLAND Collaboration, K. Eguchi et al., Phys. Rev. Lett. 90 (2003) 021802; T. Araki et al., Phys. Rev. Lett. 94 (2005) 081801.

[3] SNO Collaboration, Q. R. Ahmad et al., Phys. Rev. Lett. 89 (2002) 011301; 89 (2002) $011302 ; 92$ (2004) 181301; B. Aharmim et al., Phys. Rev. C 72, (2005) 055502.

[4] F. Pisano and V. Pleitez, Phys. Rev. D 46 (1992) 410; P. H. Frampton, Phys. Rev. Lett. 69 (1992) 2889; R. Foot, O. F. Hernandez, F. Pisano and V. Pleitez, Phys. Rev. D 47 (1993) 4158.

[5] M. Singer, J. W. F. Valle and J. Schechter, Phys. Rev. D 22 (1980) 738; R. Foot, H. N. Long and Tuan A. Tran, Phys. Rev. D 50 (1994) 34(R); J. C. Montero, F. Pisano and V. Pleitez, Phys. Rev. D 47 (1993) 2918; H. N. Long, Phys. Rev. D 54 (1996) 4691; 53 (1996) 437.

[6] P. V. Dong, H. N. Long, D. T. Nhung and D. V. Soa, Phys. Rev. D 73 (2006) 035004.

[7] P. V. Dong, H. N. Long, and D. V. Soa, Phys. Rev. D 73 (2006) 075005.

[8] P.V. Dong, H. N. Long, and D. T. Nhung, Phys. Lett. B 639 (2006) 527.

[9] P.V. Dong, Tr.T. Huong, D.T. Huong and H. N. Long, Phys. Rev. D 74 (2006) 053003.

[10] For experimental projects in preparation, see S. R. Elliott, Nucl. Phys. B. Proc. Suppl. 138 (2003) 275.

[11] J. Schechter and J. W. F. Valle, Phys. Rev. D 25 (1982) 2951, C. O. Escobar and V. Pleitez, Phys. Rev. D 28 (1983) 1166.

[12] V. Pleitez and J. M. D. Tonasse, Phys. Rev. D 48 (1993) 5274.

[13] Mohapatra and J. D. Vergados, Phys. Rev. Lett. 47 (1981) 1713.

[14] W. C. Haxton, S. P. Rosen, and G. J. Stephenso, Phys. Rev. D 26 (1982) 1805.

[15] C. O. Escobar and V. Pleitez, Phys. Rev. D 28 (1983) 1166.

[16] R. Foot, O. F. Hernandez, F. Pisano and V. Pleitez, Phys. Rev. D 47 (1993) 4158.

[17] J. C. Montero , C. A. de Pires, and V. Pleitez, Phys. Rev. D 64 (2001) 096001.

[18] Alex G. Dias at al, Phys. Rev. D 72 (2005) 035006.

[19] P. V. Dong, H. N. Long, and D. V. Soa, Phys. Rev. D 75 (2007) 073006.

[20] D. Chang and H. N. Long, Phys. Rev. D 73 (2006) 053006; M. B. Tully and G. C. Joshi, Phys. Rev. D 64 (2006) 011301.

[21] G. B. Gelmini and M. Roncadelli, Phys. Lett. B 99 (1981) 411; S. Bertolini and A. Santamaria, Nucl. Phys. B 310 (1988) 714; Y. Chikashige, R. N. Mohapatra and R. D. Peccei, Phys. Lett. B 98 (1981) 265; Phys. Rev. Lett. 45 (1980) 1926; D. Chang, W. Y. Keung and P. B. Pal, Phys. Rev. Lett. 61 (1988) 2420.

[22] W. -M. Yao et. al. (Particle Data Group), J. Phys. G: Nucl. Part. Phys. 33 (2006) 478.

[23] S. Bertolini and A. Santamaria in Ref. [21].

[24] G. B. Gelmini and M. Roncadelli in Ref. [21].

[25] M. C. Gonzalez- Garcia and Y. Nir, Phys. Lett. B 232 (1989) 383.

[26] A. Ali, A. V. Borisov and D. V. Zhuridov, help/060672.

[27] D. V. Soa, T. Inami and H. N. Long, Eur. Phys. J. C, 34 (2004) 285.

Received 28 December $200 \%$. 
